\title{
Estrategias de supervisión aplicadas por la dirección y sus implicaciones en el cumplimiento de labores del personal docente de los Colegios Técnicos Profesionales Ricardo Castro Beer y San Mateo
}

\author{
Supervision strategies applied by the principal and its implications for the fulfillment of teaching crew of the \\ Technical High Schools Ricardo Castro Beer and San Mateo
}

Recibido 20 mayo 2013 • Aceptado 24 junio 2013 • Corregido 26 junio 2013

\author{
Jesusita Triana Mora' \\ Ministerio de Educación Pública, \\ San José, Costa Rica \\ trianamora@yahoo.es
}

\begin{abstract}
Resumen. La presente investigación analiza las estrategias de supervisión que aplican los profesionales en administración de la educación del Colegio Técnico Profesional Ricardo Castro Beer y el Colegio Técnico Profesional San Mateo, en Orotina Alajuela, en función de lo que implican para el desempeño laboral del personal docente. Para ello, se trazan como objetivos específicos el identificar el proceso de supervisión utilizado por la dirección; determinar las estrategias de supervisión utilizadas y establecer el impacto de la supervisión sobre del cumplimiento de la labor docente.

La investigación se realiza dentro del marco de un estudio cuantitativo, con la participación del personal docente y profesionales en administración de la educación de las instituciones mencionadas. Los hallazgos encontrados manifiestan la ausencia de planificación, seguimiento y evaluación como etapas del proceso de supervisión que lleva a cabo la dirección en los centros educativos estudiados; ausencia de mecanismos de control como estrategias de supervisión a aplicar al personal docente; inestable fomento de las relaciones interpersonales; escasa colaboración de los profesionales en administración de la educación en el proceso de enseñanza y aprendizaje; incidencia de la supervisión ejercida por los directores en el cumplimiento del desempeño de la labor docente. Para mejorar el proceso de gestión de la supervisión en los centros educativos, se propone un modelo estratégico con acciones específicas, en función del historial de la labor docente, la participación del personal docente y la población estudiantil, para la evaluación respectiva de la supervisión docente.
\end{abstract}

Palabras clave. Educación, administración de la educación, gestión de la educación, proceso de supervisión, estrategias de supervisión, desempeño docente.

\footnotetext{
Máster en Ciencias de la Educación con énfasis en Administración Educativa de la Universidad de Costa Rica (UCR). Licenciada en Ciencias de la Educación con énfasis en Administración de la Educación de la Universidad de Costa Rica (UCR). Profesorado y Bachiller en Educación Primaria de la Universidad de Costa Rica (UCR). Licenciatura en I y II Ciclo de la Universidad Castro Carazo. Ha labora como docente de educación primaria y asistente de Dirección Regional para el Ministerio de Educación Pública (MEP). Actualmente se desempeña como docente de primaria en la Escuela Primo Vargas, en Orotina, Alajuela.
} 


\begin{abstract}
This research analyzes the monitoring strategies applied by professionals in education administration at Ricardo Castro Beer Technical High School and San Mateo Technical High School located in Orotina, Alajuela, depending on the implications for the performance of the work of teachers. For this purpose, specific objectives are plotted to identify the monitoring process used by the directors; determine the strategies used in the work of supervision and establish the implications of supervision conducted in compliance function of teaching.

This research is conducted within the framework of a quantitative study, with the participation of teachers and principals of the institutions mentioned. The findings show the absence of planning, monitoring and evaluation and monitoring stages of carrying out the direction studied in schools, lack of control mechanisms and monitoring strategies to apply to teachers; unstable building interpersonal relationships; little help from other professionals in education administration in the teaching and learning process, and impact of the supervision exercised by the directors in fulfilling the teaching performance. To improve the process monitoring management in schools, a strategic model with specific actions is proposed, depending on the history of teaching, participation of teachers and the student population for the respective assessment of teacher supervision.
\end{abstract}

Keywords. Education, educational administration, educational management, process monitoring, supervision strategies, teacher performance.

\title{
Introducción
}

La educación es la herramienta primordial para la inserción en la sociedad actual. El proceso de aprehensión de educación formal es complejo, dinámico y específico de cada contexto. Lo particular es que cada sistema y por ende, cada centro educativo, se enfoca en alcanzar la calidad en la educación y si la organización no se interesa en ello, la comunidad así lo exige. Al respecto, Gento (2008) manifiesta:

Los identificadores de la calidad institucional de un centro educativo son aquellos componentes que permiten valorar la medida en que dicha institución alcanza los niveles de calidad en sus resultados. Hemos estructurado tales componentes en torno a: producto educativo, satisfacción de los alumnos, satisfacción del personal, impacto de la educación. (p. 29)

La calidad de la educación no posee definición propia ni estable; ella representa el grupo de valores y expectativas de cada sociedad. Al respecto, Garbanzo (2002) afirma:

La calidad de la educación es un elemento contextual, es decir cada situación, cada momento histórico, cada proyecto educativo presenta un concepto de calidad educativa. No hay una definición general del concepto de calidad de la educación, se define según los paradigmas de acción de la educación. (p. 92)

En concordancia con Garbanzo (2002), los paradigmas son propios de cada organización educativa; lo que motiva a la elección de esta temática. Una de las problemáticas que afecta 
en los centros educativos son los constantes comentarios realizados por el estudiantado, docentes, padres y madres de familia, así como la comunidad, en general, sobre el abuso de poder. Además, la referencia al incumplimiento tanto en las normas éticas como laborales de algunos docentes en los centros educativos. Este ambiente ha provocado desmotivación y hasta deserción estudiantil, así como roces dentro del clima laboral.

Es función de la administración de la educación, velar por todas las irregularidades que sucedan dentro de la institución, tal como lo afirman García, Rojas y Campos (2002), cuando refieren que:

El rol del director está comprometido con el proceso de conducción de la escuela, entendido este como un metaproceso que imprime una determinada dirección u orientación global a la organización, involucra otros procesos, en particular el de enseñanza y aprendizaje, y opera como su facilitador u obstructor. (p. 21)

Desde esta perspectiva, la investigación que se realiza tiene importancia, puesto que se centra en la gestión que aplican los profesionales en administración de la educación, en función de lo mencionado, sobretodo, el desempeño laboral del personal docente. A través de la supervisión de la labor docente, es que la administración de la educación podrá encontrar elementos esenciales para aprovechar o fortalecer el proceso de enseñanza aprendizaje, para de alcanzar el nivel de calidad educativa que se estipula en el centro educativo.

No hay duda de que el punto de apoyo fundamental para la potenciación de la calidad educativa de un centro escolar radica en los profesores, que han de ser considerados como los promotores más directos de la misma. (Gento, 2008, p. 33)

Resulta entonces vital, conocer y analizar el proceso de supervisión ejercido por la administración del centro educativo; tomando en cuenta que la supervisión es la acción que permite controlar todos los aspectos relacionados con la educación y lo que ocurre en su entorno; es también una acción que genera indisposición en la parte que es supervisada.

Por su parte, Fernández y Gutiérrez (2005) expresan que:

En la profesión docente aumentan el estrés, la sensación de ansiedad y las enfermedades profesionales en idéntica medida en que aumentan las demandas sociales sobre el sistema educativo o en la misma proporción en que las administraciones tienden a aumentar las responsabilidades y necesidades de preparación de los profesores ante las reformas, sin que ello signifique contrapartidas en forma de reconocimiento social, económico o profesional. (p. 218)

Es decir, el solo hecho de ser docente representa un alto nivel de estrés y si, aunado a lo anterior, hay carencia de convicción en la labor, se puede caer en el abuso de poder por parte 
del personal docente hacia el alumnado. En ese sentido, Álvarez y López (1999) afirman que:

La función docente no está exenta tampoco del elemento "control" entendido como "poder" en el más amplio sentido de la palabra. Educar y enseñar no es otra cosa que influir, dirigir y orientar la mente...Desde esta perspectiva, el profesor tiene un enorme poder y lo ejerce de forma inconsciente sobre el alumno. (p. 13)

Este poder, ejercido por los docentes, puede ser amenazante para el debido cumplimiento de las labores y el trato con la población estudiantil, por lo que es importante la existencia de controles dirigidos hacia el desempeño de la labor docente, lo que ocurre a través de la supervisión del profesional en administración de la educación.

La investigación se realiza con el aval de los profesionales en administración de la educación del Colegio Técnico Profesional Ricardo Castro Beer y el Colegio Técnico Profesional San Mateo, ambos ubicados en Orotina, Alajuela, Costa Rica, con el fin de abordar el proceso de supervisión que aplican para controlar el cumplimiento de labores, la opinión que tiene el personal docente sobre la administración y el cumplimiento de labores de sus colegas, así como la valoración que tienen los docentes ante la comunidad estudiantil, dado que la población estudiantil es la razón de ser de la educación.

A partir de las interrogantes planteadas, se establecen los siguientes objetivos de investigación.

\section{Objetivos generales}

- Analizar las estrategias de supervisión que aplica la administración del CTP Ricardo Castro Beer y CTP de San Mateo y sus implicaciones en el cumplimiento de labores del personal docente

- Proponer una herramienta de gestión para fortalecer la supervisión de la labor del personal docente en el CTP Ricardo Castro Beer y CTP de San Mateo.

\section{Objetivos específicos}

- Identificar el proceso de supervisión para la labor docente que lleva a cabo el profesional en administración de la educación del CTP Ricardo Castro Beer y CTP de San Mateo.

- Determinar las acciones de supervisión que utiliza la administración de la educación del CTP Ricardo Castro Beer y CTP de San Mateo, en relación con la labor docente.

- Establecer las implicaciones de las estrategias de supervisión que aplica el profesional en administración de la educación en relación con el cumplimiento de labores del personal docente, en el CTP Ricardo Castro Beer y CTP de San Mateo.

- Diseñar un instrumento para la gestión de la supervisión del cumplimiento de labores por parte del personal docente, en el CTP Ricardo Castro Beer y CTP de San Mateo. 


\section{Marco teórico}

La investigación se sustenta en el desarrollo de los conceptos teórico-conceptuales: administración de la educación, gestión de la educación, supervisión de la educación, principios de supervisión educativa, estrategias de supervisión; así como los deberes y obligaciones en el cumplimiento de labores docentes.

\section{Administración de la educación}

La administración de la educación es la disciplina encargada de la acción educativa en los centros escolares, de ahí que incurre en la acción de educar. Los profesionales en administración de la educación, como líderes, están ligados estrechamente con las reformas, el dictamen de parámetros que controlan y fiscalizan desde los altos mandos; estos a su vez, delegan funciones hacia las líneas jerárquicas inferiores como las regionales, circuitos escolares hasta llegar a los centros educativos.

Una organización es un grupo estructurado que se rige por normas, reglamentos, procedimientos, rutinas y otros aspectos necesarios para establecer las relaciones entre los órganos, cargos y funcionarios, con el fin de mantener el equilibrio y alcanzar los objetivos (Chiavenato, 2006).

En ese sentido, las organizaciones educativas se consideran entes complejos en los que interactúan diferentes elementos que, coordinados, promueven el desempeño de la comunidad educativa, hacia el logro de los objetivos institucionales.

La administración de la educación focaliza la acción directiva, en función del contexto en que se encuentra la organización educativa, cuyo sistema puede entenderse como dinámico, con altas aspiraciones y en constante lucha por su autonomía institucional, con el afán de lograr los objetivos acordes con las necesidades y características de cada organización (Venegas, 2000).

Rodríguez (2006) afirma que dirigir una organización educativa, "es el proceso que realiza un administrador o líder para influir en los demás colaboradores para realizar un trabajo unido y de manera eficaz" (p.87), lo que refiere, directamente, a la incidencia que ejerce el profesional en administración de la educación sobre los diferentes procedimientos administrativos y organización de recursos, en función de las metas yestrategias definidas en el marco institucional.

Los encargados de la dirección de los centros educativos deben cumplir una importante función, la de supervisar el desempeño docente y administrativo del personal a su cargo, con el fin de mejorar las acciones que llevan a cabo, en beneficio de la población estudiantil y del servicio educativo que se brinda. 


\section{Gestión de la educación}

La coordinación de las acciones de planificación, organización, dirección y control del proceso administrativo, por medio de estrategias definidas para atender y anticipar el cambio, es la mejor referencia de lo que es la gestión de la educación.

En ese sentido, Antúnez (1993) describe la gestión como "un conjunto de acciones de movilización de recursos orientadas a la consecución de objetivos" (p.60), lo que concibe un proceso integral en la que es fundamental la participación de la comunidad educativa, con la debida orientación del profesional en administración de la educación.

La gestión de la educación, por tanto, articula el quehacer educativo de manera que tal que coincidan las distintas responsabilidades y acciones, que permiten una adecuada conducción de la organización hacia la consecución de la metas propias del centro educativo.

Uno de los factores principales que tiene que considerar el profesional en administración de la educación en su gestión, es el manejo del recurso humano, por lo que es de relevancia identificar las estrategias que inciden en las relaciones de interacción y comunicación que intervienen en "hacer que las cosas sucedan" (Blejmar, 2005).

En ese sentido, es fundamental que la gestión que lleva a cabo la dirección en relación con la labor docente, debe buscar apoyo en la motivación, promoción, negociación y comunicación, aspectos que sirven de aliciente para el desempeño satisfactorio de la labor asignada.

\section{Supervisión de la educación.}

La supervisión educativa se relaciona con aquellas labores que realizan los profesionales en administración de la educación, para controlar las acciones docentes dentro de su organización.

De acuerdo con Robbins (2004), supervisar es un acto inclusivo de control, que influye en el desempeño docente, de manera que se orienta su quehacer al mejoramiento del proceso de enseñanza y aprendizaje, en función de la formación integral del educando que tiene a cargo.

De esta manera, la supervisión educativa refiere al control, medición y asesoramiento que se realiza sobre la dinámica educativa, principalmente en la labor del personal docente. Al respecto, Garbanzo (2002) señala que “...la supervisión es una función inherente de los sistemas educativos, coadyuva con el proceso enseñanza y aprendizaje, centra su atención en el docente, facilitándole el desarrollo de su potencial personal y profesional" (p. 5).

De ahí, que se entienda la supervisión como una herramienta que tiene el objetivo de mejorar la educación, identificando los factores que caracterizan a los "buenos profesionales docentes y los buenos proyectos que funcionan" (Teixidó, 1997, p. 57), en la organización 
educativa.

El profesional en administración de la educación, al realizar la supervisión debe tener en cuenta los deberes y obligaciones del personal supervisado, sin dejar de lado otras funciones que cumple en el ejercicio de control, como es la tarea de asesorar, acompañar y mejorar el desempeño profesional.

Así, "la supervisión fundamenta, incide y condiciona la educación debe tomar en consideración todo lo optimiza la educación" (Teixidó, 1997, p. 49), por lo que debe contemplar todos los factores internos, externos, humanos, materiales, funcionales y otros, en un determinado momento.

\section{Proceso de supervisión.}

La supervisión como una de las funciones que realiza la administración de la educación, en su proceso de control, refiere la necesidad de organización institucional, con el fin de establecer el modo, tiempo, estrategias y medios que va a considerar en la evaluación. Se lleva a cabo a través de un proceso de supervisión que enfatiza la planificación, el seguimiento y evaluación del desempeño del personal, desde la optimización del cumplimiento de funciones.

El proceso de supervisión consiste básicamente, en la elaboración de un plan para supervisar las funciones de los subalternos o colaboradores.

De esta forma, se determina que el proceso de supervisión de la educación se enfoca en acciones continuas y entrelazadas que se realizan, con el fin de obtener información oportuna para la toma de decisiones, en procura de la mejora de la educación desde ámbitos diversos como calidad, administración, interrelaciones personales y contexto.

De acuerdo con Campos (2008), el proceso de supervisión se cumple en tres etapas o fases: planeamiento, seguimiento y evaluación.

La etapa de planificación del proceso de supervisión corresponde al diagnóstico y elaboración respectiva del plan, en relación con el desempeño docente. Para ello, se trata de responder al para qué se realiza la supervisión, el qué se va a supervisar, el dónde se lleva a cabo la misma, el cómo, cuándo y con qué, a fin de determinar las acciones que el personal docente ejecuta para el desarrollo del proceso de enseñanza y aprendizaje.

El diseño del plan de supervisión, consta de fases específicas que conllevan a identificar la situación educacional, los objetivos y metas establecidas, el período en que se realiza, el alcance esperado y los aspectos por supervisar, en relación con las técnicas e instrumentos, recursos y cronograma de actividades. Una vez establecida esta parte del proceso, se inicia la ejecución 
de la supervisión, considerando los indicadores anteriormente mencionados (Campos, 2008).

Se diseña el plan de trabajo que orienta la labor de control, como parte de las tareas administrativas que involucra la gestión educativa del profesional en administración de la educación.

La etapa de seguimiento corresponde a la segunda fase del proceso de supervisión, por medio del cual se realiza un rastreo de la actuación docente, en función de las técnicas y métodos aplicados.

En forma general, el procedimiento de seguimiento, según Campos (2008), consta de:

- Entrevista inicial: primer contacto de la persona supervisora con el personal supervisado. Su principal objetivo es lograr un ambiente de confianza que permita informar aspectos como: objetivo de la visita, metodología a aplicar y cronograma de trabajo.

- Recolección de información: consiste en la realización de observaciones y técnicas e instrumentos utilizados para recoger los datos más relevantes del desempeño docente.

- Entrevista de salida: la persona supervisora se reúne nuevamente con el personal supervisado, para presentar el informe respectivo, en el que se consideran aspectos positivos y deficitarios observados durante el proceso de supervisión. En esta entrevista, se puede interactuar aportando comentarios y sugerencias para mejorar.

La tercera etapa del proceso de supervisión, corresponde a la aplicación de mecanismos de control que permiten una evaluación continua del desempeño docente, con el fin de otorgar informes de realimentación que enriquezcan la labor profesional y la toma de decisiones al respecto.

Esta fase comprende, por tanto, la esencia misma del proceso de supervisión, por lo que reviste especial importancia al respecto.

La supervisión es un proceso de confianza y participación activa de las partes involucradas, por lo que requiere estrategias precisas y pertinentes con la labor que se realiza, en función del mejoramiento del proceso de enseñanza y aprendizaje.

Con el afán de fructificar el proceso de evaluación, Campos (2008) señala que es importante:

- La elaboración y presentación de un informe ordenado y coherente, de acuerdo con los aspectos supervisados, los problemas identificados, así como los acuerdos y sugerencias.

- El aporte de datos referenciales, criterios supervisados, apreciación sobre el avance, las conclusiones y recomendaciones determinadas; además de los anexos pertinentes.

- La retroalimentación de parte de la persona que supervisa a quien es supervisado, con 
el afán de obtener la aceptación de la evaluación, la debida orientación y asesoramiento requerido, la búsqueda de soluciones en relación con las deficiencias, la verificación del cumplimiento delas recomendacionesy sugerencias, asícomoel reajustey reprogramación de acciones, para el logro de los objetivos y metas.

Lo anterior, contribuye al fortalecimiento de la labor docente, en relación con el cumplimiento de sus funciones, a través de la supervisión ejercida por la dirección en términos de la gestión curricular y pedagógica que se realiza en torno al proceso de enseñanza y aprendizaje.

\section{Estrategias de supervisión.}

Las acciones que se realizan forman parte de una secuencia planificada, de manera sistemática y secuencial, para un determinado fin, lo que se conoce como estrategia.

Al respecto, Arroyo (2000) define la estrategia como una guía de acciones que deben seguirse para alcanzar objetivos en las organizaciones educativas, por lo que tienen una clara intención definida. métodos:

Nérici (1986) citado por Romero (2001), clasifica la acción de la supervisión en los siguientes

Método científico, en el cual se observa al personal docente en el desempeño de sus funciones, utiliza una lista de cotejo para registrar las acciones que lleva a cabo el docente.

Método no directivo, cuyo fin pretende que el docente reflexione sobre sus errores, tome conciencia y encuentre soluciones a las fallas cometidas en el desempeño de sus funciones.

Método de facetas múltiples o mixtas, se planifican diferentes técnicas de supervisión según el caso que en que se esté llevando a cabo el proceso. La combinación de técnicas es esencial en este método.

Método de ayuda mutua o interpersonal, en el que se da la integración de la persona supervisora y el docente que se supervisa.

Método clínico, cuya función permite orientar al docente para que supere las deficiencias demostradas y prevenir para que no incurra en otras.

Método por ósmosis, el cual promueve experiencias pedagógicas mediante el intercambio de metodologías, experiencias y técnicas.

Método de investigación activa, en el que se realiza un estudio en grupo, por medio de 
reuniones periódicas para analizar situaciones precisas.

Método de micro enseñanza, se proveen recursos para el mejoramiento didáctico del docente.

Método Delphi, consta de estrategias para consensuar entre los participantes del proceso, con el fin de lograr un acuerdo entre ellos.

Método de representación operativa, se escenifica una situación real a cargo de especialistas con el afán de analizar, reflexionar y mejorar sobre la labor docente.

Método descriptivo de escena, pedir al personal docente que manifieste por escrito su opinión acerca de un hecho propuesto.

\section{Técnicas de supervisión educativa.}

A partir de los métodos mencionados, se determinan diferentes acciones que especifican las formas de llevar a cabo el proceso de supervisión, a través de técnicas que puede aplicar el profesional en administración de la educación, en función de la labor del personal docente.

Desde esta perspectiva, las técnicas de supervisión Gento (2008) se encuentran: la abierta o informal, la evaluativa, la clínica y la participativa. Cada una de ellas, responde a las necesidades propias del proceso de supervisión, de acuerdo con la persona o situación que amerite el seguimiento.

Supervisión abierta o informal: no se establecen programas o anuncios de las visitas que se realizan, se presta atención a los detalles o situaciones que surjan para un conocimiento más fidedigno de la realidad. La comunicación que se da entre la persona que ejerce la supervisión y la supervisada, ocurre de un modo fluido, lo que permite, además:

(...) dar oportunidad a aquellos sobre quienes se incide a que se exprese con libertad: deben, incluso, provocarse situaciones en las que esto se produzca (tales como reuniones informales para escucharles, sesiones de claustro abiertas, contactos equivalentes con el equipo directivo, con los profesores, con los alumnos, con los padres, etc.). (Gento, 2008, p.196)

Esta técnica de supervisión se orienta a la búsqueda de solución de problemas y no a la de culpables; por lo que media la responsabilidad profesional que asume la persona supervisora de colaborar con el personal docente a su cargo, en el desarrollo del proceso de enseñanza y aprendizaje.

Supervisión evaluativa: tiene la finalidad de ejercer un control sobre el manejo de bienes 
a cargo, en este caso, del personal docente para el ejercicio de sus funciones. Se realizan acciones como el estudio de documentos, datos o hechos relacionados con la labor docente, con el fin de conocer la realidad en que desempeña su labor y el grado de compromiso para mejorar en ella.

Supervisión clínica: asigna como tarea dictaminar la forma de mejorar la calidad de la educación, por lo que pretende encontrar debilidades presentes en el planeamiento educativo.

Al respecto, Gento (2008) manifiesta que "la supervisión clínica tiene un enfoque esencialmente orientado hacia la mejora de la educación y del funcionamiento de los responsables y las instituciones en que se lleva a cabo" (p. 200).

Incide directamente en la población estudiantil y sirve de apoyo al docente en su labor, mediante la adaptación curricular correspondiente a las necesidades y requerimientos de cada quien.

Supervisión participativa: se orienta a la realización de una supervisión en la que, tanto las personas supervisoras como las supervisadas, participan en la toma de decisiones para el mejoramiento del desempeño docente. Es tarea de la persona supervisora velar porque el grupo de personas que participen de la actividad docente para que muestren interés en lo que hacen, disposición de mejora y trabajo en equipo, actitud de respeto, tolerancia, pluralismo, ideológico y libre expresión de ideas, otras.

\section{Cumplimiento de labores del personal en el ámbito de la educación pública costarricense.}

La labor docente ocurre en todo momento y lugar de la organización educativa, puesto que incluye desde la preparación de lecciones, hasta el desarrollo de pruebas y otros documentos que deben mantenerse al día, actualizados. En ese sentido, el Servicio Civil (2004) elabora el Manual Descriptivo de Clases de Puestos Docentes, en el que se especifican las funciones del personal docente, considerando su comportamiento social y la forma de interrelacionarse con la comunidad educativa.

Las acciones de la labor docente, en la educación secundaria, son las siguientes:

- Planear, preparar e impartir lecciones de acuerdo con su especialidad.

- Preparar materiales didácticos para uso en sus lecciones, a través de diferentes estrategias metodológicas.

- Confeccionar, aplicar y calificar pruebas de evaluación que permiten medir el conocimiento de la población estudiantil. 
- Ejecutar acciones docentes a partir de las políticas educativas y otras orientaciones establecidas por el Ministerio de Educación Pública.

- Respetar las diferencias individuales de las personas, especialmente de su estudiantado, considerando su credo, lugar de procedencia, costumbres, necesidades educativas, entre otros.

- Participar en actividades cívicas y educativas que organiza la institución educativa, con el fin de colaborar en el proceso de enseñanza y aprendizaje y la motivación de sus estudiantes; además de fortalecer la cultura y convivencia organizacional.

- Participar en actividades sociales institucionales que se organicen, en beneficio del centro educativo, mientras no se atente contra sus principios morales.

- Atender las inquietudes familiares en relación con el proceso de aprendizaje del estudiantado, con el fin de brindar la orientación adecuada y la información pertinente al respecto.

- Actualizar constantemente los documentos, libros, listas y registros que conlleva su acción docente en el aula y en la institución educativa.

- Manifestar un comportamiento acorde con las normas disciplinarias que plantea la organización educativa.

- Buscar la excelencia en el desarrollo de su labor docente, mostrando interés en las posibilidades de superación, en nuevas herramientas y oportunidades que se presentan, entre otros, para el mejoramiento de la calidad de su desempeño profesional.

- Ser responsable del desarrollo de su labor, en función del cumplimiento de la puntualidad en su horario, en la entrega de documentación a la dirección, en la devolución de trabajos calificados a la población estudiantil, entre otros.

- Aplicar los principios éticos que se relacionan con su labor profesional, por medio de los cuales mantiene una imagen decorosa, utilizael lenguaje adecuado, se dirigecorrectamente a la comunidad educativa, realiza su trabajo con esmero y dedicación, entre otros.

- Manifestar un trato cortés y satisfactorio en relación con la comunidad educativa, basada en el respeto y buena actitud.

- Actualizar los conocimientos y técnicas docentes de manera continua, a través de la asistencia a sesiones de actualización para su desempeño profesional.

Corresponde al profesional en administración de la educación, conocer las características laborales y actitudinales del personal docente a su cargo, para lograr un equipo de trabajo que responda a las necesidades, intereses y características de la organización educativa. De ahí, la relevancia de la supervisión docente como parte de la gestión de la educación que se lleva a cabo, la cual vela por el desarrollo de las acciones curriculares y administrativas del centro 
educativo, en función del proceso de enseñanza y aprendizaje que aplican los docentes, a quienes orienta, asesora y evalúa en el ejercicio de su labor.

\section{Marco metodológico}

La presente investigación se considera como un estudio de corte cuantitativo. Lo anterior, dado que utiliza la recolección de datos y la medición numérica para la realización de un análisis estadístico que permite el establecimiento de patrones de comportamiento en el contexto en el que se lleva a cabo el proceso (Hernández, Fernández y Baptista, 2010, p.4).

Tanto la recolección de datos como el análisis de la información, se trata de manera objetiva, conforme a la realidad que se estudia, por lo cual se enfoca en la descripción detallada de las pesquisas encontradas.

Al respecto, Hernández, Fernández y Baptista (2010), mencionan que los estudios descriptivos busca la determinación de propiedades, características y rasgos importantes del fenómeno que se analiza, con el fin de narrar las situaciones y manifestaciones propias del grupo que participa, en el tiempo en el que se lleva a cabo la investigación.

Los sujetos participantes en el estudio están conformados por el personal docente de los colegios técnicos profesionales Ricardo Castro Beery San Mateo; además de los dos profesionales en administración de la educación de ambas instituciones.

La recolección de datos se realiza en tres momentos: el primero, que corresponde al ingreso a cada una de las instituciones para solicitar el consentimiento de los directores en relación con la aplicación del instrumento; el segundo, la visita para entregar el cuestionario al personal docente y hacer la entrevista a los profesionales en administración de la educación; el tercer momento, la recogida de los instrumentos con la información suministrada por los docentes.

Para la recolección de información en esta investigación, se utilizan como instrumentos el cuestionario y la entrevista estructurada.

El cuestionario se aplica al personal docente, por lo que consta de una serie de preguntas cerradas y respuestas establecidas por medio de una escala Likert, para conocer la opinión de los participantes.

\section{Análisis de la información}

El análisis de los datos recolectados, se segmenta tomando como parámetro las categorías de análisis y sus indicadores, a partir de los objetivos específicos establecidos para el proceso de 
investigación.

\section{Caracterización de los participantes}

La caracterización de los participantes, conformados por la dirección y el personal docente del Colegio Técnico Profesional Ricardo Castro Beer y Colegio Técnico Profesional de San Mateo, refiere criterios como condición laboral, grado académico y años de laborar en la institución educativa. En el caso de los profesionales en administración de la educación, se agrega el criterio de años de servicio en la dirección, con el fin de determinar su experiencia en el área.

Así, el personal docente, presenta la siguiente información.

Tabla 1

Caracterización del personal docente del Colegio Técnico Profesional Ricardo Castro Beery San Mateo Nota: Cuestionario aplicado al personal docente del CTP Ricardo Castro Beer y San Mateo, 2012.

En relación con los profesionales en administración de la educación, los directores de las organizaciones educativas se destacan por:

\begin{tabular}{cll}
\hline Criterio & \multicolumn{1}{c}{ CTP Ricardo Castro Beer } & \multicolumn{1}{c}{ CTP San Mateo } \\
\hline Condición laboral & 50\% propiedad & $73 \%$ propiedad \\
& $50 \%$ interino & $27 \%$ interino \\
Grado académico & 21\% Bachillerato & $9 \%$ Bachillerato \\
& $50 \%$ Licenciatura & $91 \%$ Licenciatura \\
Años en la & 29\% Maestría & \\
institución & 29\% menos de un año & $9 \%$ menos de un año \\
& 14\% entre tres y cinco años & $9 \%$ entre uno y tres años \\
& $29 \%$ entre cinco y diez años & $18 \%$ entre tres y cinco años \\
& & $5 \%$ entre cinco y diez años \\
& & $9 \%$ más de diez años \\
\hline
\end{tabular}

- Laborar en propiedad.

- Poseer un grado académico de maestría.

- Tener de cinco a diez años de laborar en la institución.

- Poseer una experiencia entre cinco y diezaños o más de laborar en elárea de administración de la educación. 


\section{Proceso de supervisión de la labor docente.}

La educación, como proceso formativo, está conformada por una serie de etapas que presuponen el perfeccionamiento de las personas que la reciben, quienes buscan comprender y transformar la realidad en la que están inmersos, a través de la participación en diferentes escenarios educativos de calidad.

Para cumplir con ese cometido, se lleva a cabo una función supervisora de la educación, en función de la labor docente, por medio de la cual se da un seguimiento, control y evaluación de su desempeño en la organización educativa. Esto permite el acompañamiento, apoyo y asistencia por parte de la dirección, con el fin de mejorar la calidad del proceso de enseñanza y aprendizaje.

En ese sentido, la supervisión es un acto inclusivo de control que influye, directamente, en el desempeño docente, de manera que orienta su quehacer al mejoramiento del proceso de enseñanza y aprendizaje, en función de la formación integral del educando que tiene a cargo (Robbins, 2004).

Por ello, se entiende como el proceso que permite prever e identificar situaciones que ameritan la atención docente, con el fin de encontrar soluciones que benefician la dinámica institucional y, por ende, el proceso de aprendizaje de la población estudiantil.

De esta manera, se analiza el proceso de supervisión de la labor docente que se realiza en el CTP Ricardo Beer y CTP San Mateo, en Orotina, de acuerdo con las etapas que define Campos (2008): planeamiento, seguimiento y evaluación, de las cuales se manifiestan los siguientes resultados:

- En relación con la etapa de planeamiento: Solo en algunas ocasiones se indica la precisión de la razón de ser de la supervisión, nunca se lleva a cabo un diagnóstico de necesidades del personal.

- En relación con la etapa de seguimiento: Nunca se lleva a cabo la presentación de un cronograma para la ejecución de la supervisión, no se realiza una entrevista inicial con el docente; solo algunas veces se define la metodología a seguir.

- En relación con la etapa de evaluación: Nunca se realiza un informe sobre lo supervisado para cada docente, ni se da una realimentación al personal docente sobre aspectos importantes identificados. Tampoco se crean planes de mejora en coordinación con el docente supervisado, lo que puede incidir en el desempeño profesional del educador o educadora, ni se reorienta el desarrollo de las actividades docentes en función de 
los resultados obtenidos en el proceso de supervisión. Nunca se informa al personal docente sobre los progresos logrados según los objetivos planteados en el proceso de supervisión realizado.

De esta manera, la gestión del proceso de supervisión conlleva la consideración de los aspectos anteriormente mencionados, por medio de los cuales el profesional en administración de la educación puede desarrollar un trabajo conjunto con el personal docente de control constante de las funciones que realiza. Con ello, brinda la asistencia requerida por los docentes y aplica una serie de métodos y técnicas de supervisión, que conforman estrategias pertinentes para el perfeccionamiento de la labor docente.

\section{Estrategias de supervisión}

El proceso de supervisión se realiza a partir de una serie de acciones organizadas, de manera sistemática y secuencial, que permiten la consecución de los objetivos y metas establecidos en la organización, en beneficio del proceso de enseñanza y aprendizaje.

Al respecto, Arroyo (2000) se refiere a las estrategias como la guía de acciones para alcanzar un objetivo, con el fin de prevenir y rectificar el desarrollo de las funciones docentes, en el contexto educativo en que se desenvuelven.

En este sentido, el profesional en administración de la educación, por medio de la utilización de diferentes métodos y técnicas de supervisión, pretende alinear la organización desde el mejoramiento del desempeño de cada uno de los miembros de la comunidad educativa, en la cual el docente adquiere un papel relevante.

De ahí, que Bartle (2010) considera que las estrategias para el proceso de supervisión enfatizan el análisis del desempeño docente, los aportes que hace al centro educativo, la identificación de problemas en el cumplimiento de la labor docente para la búsqueda de soluciones asertivas, el aseguramiento del desarrollo adecuado del proceso de enseñanza aprendizaje y demás responsabilidades a su cargo, utilización de la experiencia docente y la realimentación en la acción supervisora ejercida por la gestión de la educación.

De esta manera, las estrategias se establecen para la intervención docente, conforme con las condiciones pertinentes para la toma de decisiones, en función del cumplimiento de labor de los educadores y educadoras, con el fin de ajustar y modificar el comportamiento y grado de compromiso con la organización educativa.

Para efectos de esta investigación, se agrupan las estrategias de supervisión docente en: aquellas que sirven como mecanismos de control para el ejercicio docente, aquellas que 
refuerzan las relaciones interpersonales y que, por tanto, enriquecen el desarrollo de la labor docente; y aquellas que colaboran en el proceso de enseñanza aprendizaje.

En cuanto al control, se considera como función dinámica para el aseguramiento del buen desempeño docente, por lo que implica una inspección profesional que garantiza el cumplimiento de la labor asignada, por medio de la cual se evalúan las decisiones que fueron tomadas en el momento oportuno, de acuerdo con criterios establecidos para tal fin. De ahí, la importancia de razonar su uso en el proceso de supervisión para el control del desempeño docente, como menciona Nérici (1986) citado por Romero (2001).

De esta manera, los mecanismos de control como estrategias de supervisión, inciden en la modificación del comportamiento docente en el desarrollo de sus funciones, para verificar la eficiencia y eficacia de la actividad que se realiza en el centro educativo.

Las estrategias de supervisión de la labor docente relacionadas con las relaciones interpersonales incentivan la interacción recíproca entre las personas en la organización educativa, por lo que promueve una serie de acciones que permiten la apertura al diálogo asertivo entre las partes que participan en el desarrollo de la inspección que se realiza. Así, "las relaciones interpersonales consisten en la interacción recíproca entre dos o más personas, involucrando los siguientes aspectos: la habilidad para comunicarse efectivamente, el escuchar, la solución de conflictos y la expresión auténtica" (López, 2011, p.8).

De esta forma, la profesión docente requiere de un desempeño social que permita el intercambio fluido de técnicas pedagógicas, administrativas y otras que favorezcan el cumplimiento de la labor en las organizaciones educativas, mediante la participación activa de todos los actores involucrados en el acto educativo.

Asimismo, las acciones de supervisión que colaboran en la enseñanza aprendizaje, pretenden contribuir al perfeccionamiento del proceso, en función de los objetivos organizacionales planteados, por lo que es una necesidad permanente en el centro educativo.

Es así como, Cambronero (2002), menciona que la supervisión es una función formativa que no solo trata la comprobación de errores y su proceso de superación; sino que refiere más a proceso de colaboración, orientación y coordinación entre docentes y la dirección, de manera que se brinde una participación más directa para el mejoramiento de la enseñanza y el aprendizaje.

A partir de las consideraciones anteriores, se expresan los resultados emitidos por los sujetos participantes en el estudio, personal docente y profesionales en administración de la 
educación, en cuanto a las estrategias de supervisión que aplica la dirección. A saber:

- En relación con las estrategias como mecanismos de control: Algunas veces se realizan observaciones al personal docente, nunca se utilizan listas de cotejo y escalas para medir las acciones docentes.

- En relación con las estrategias para el fomento de las relaciones interpersonales: Siempre se utiliza el diálogo como medio de comunicación con el personal docente; además del uso de una comunicación fluida y clara con la dirección. También siempre se busca el consenso entre el personal docente, para la toma de decisiones relacionadas con su labor.

- En relación con las estrategias para la colaboración en el proceso de enseñanza y aprendizaje: Nunca se coopera con el personal docente en el fortalecimiento del proceso de enseñanza aprendizaje.

De ahí, que la supervisión sea una acción dinámica en la gestión que lleva a cabo la dirección, en el centro educativo, puesto que le permite conocer la labor docente, a fin de ayudar y orientar el desarrollo de sus funciones. Las acciones referentes al proceso de enseñanza y aprendizaje, deben promover apoyos pedagógicos que le permitan al docente, adaptarse y progresar en su desempeño.

En ese sentido, la colaboración se presenta como la mejor herramienta en la supervisión para el mejoramiento del proceso de enseñanza y aprendizaje que se desarrolla en las organizaciones educativas.

\section{Implicaciones del proceso de supervisión en el cumplimiento de la labor docente}

El cumplimiento del deber y obligaciones inherentes al cargo, permite al personal docente el ejercicio de un buen desempeño en las organizaciones educativas.

En ese sentido, es una tarea relevante para la administración de la educación, considerar acciones que permitan el establecimiento de un sistema de medición del desempeño, en el nivel individual y organizacional, con el fin de encauzar la acción en los diferentes procesos educativos que se desarrollan (Gutiérrez, 2006).

Lo anterior, permite la toma de conciencia de la actuación docente para una búsqueda de mejoramiento constante, de manera eficiente, lo que incentiva su compromiso con la institución.

Para el análisis de las implicaciones del proceso de supervisión en el cumplimiento de la 
labor del personal docente, en el CTP Ricardo Beer y CTP San Mateo, referente al proceso de enseñanza y aprendizaje, las relaciones interpersonales y el desempeño profesional que se lleva a cabo. Así:

- En relación con las implicaciones en el proceso de enseñanza aprendizaje: Siempre la supervisión incide en el mejoramiento en la planificación y preparación de lecciones y en la preparación y utilización de materiales didácticos, por parte del personal docente. Además, siempre los docentes confeccionan pruebas conforme las características de la población estudiantil. También se indica que siempre se ejecuta la política educativa en sus acciones docentes

- En relación con las implicaciones en las relaciones interpersonales: Siempre se muestra respeto por las diferencias individuales de sus estudiantes y se da la colaboración entre los compañeros de trabajo. Además, siempre se atienden las inquietudes de los padres y madres de familia.

- En relación con las implicaciones en el desempeño profesional: Siempre se da la actualización de documentos, libros, listas y registros, por parte del personal docente, quienes cumplen con las normas disciplinarias en el ejercicio de su labor. Además, siempre se busca la excelencia en su labor, lo que proporciona un buen desempeño docente, lo que indica que el personal se responsabiliza de su labor. También, se evidencia la aplicación de la ética profesional en la labor educativa del personal docente; asimismo, siempre se actualizan los conocimientos y técnicas propias de su especialidad, de manera constante.

Desde esa perspectiva, la supervisión y el desempeño docente conllevan un trabajo conjunto que permite el constante diálogo acerca del proyecto educativo, en función de la labor docente, quien en su ejercicio profesional debe mostrar ser competente en el trato a las personas, en la convivencia grupal, la aplicación de conocimientos básicos de su profesión y el adecuado desarrollo del proceso de enseñanza y aprendizaje.

\section{Consideraciones finales}

Al concluir el análisis, se estructura una visión general de las coincidencias en la opinión manifestada por las direcciones y el personal docente de ambos colegios. Lo anterior, permite tener una panorámica de las categorías de análisis tratadas en el estudio, las cuales refieren al proceso de supervisión, las estrategias de supervisión y las implicaciones de la supervisión en el cumplimiento de labores del personal docente. 
Cada categoría, en este caso, representa el 100\%, por lo que en las figuras que se presentan se muestra un porcentaje que corresponde a cada una, basado en esa representación.

La Figura 1, muestra las coincidencias de opinión entre el personal docente y la dirección del CTP Ricardo Castro Beer, según las categorías analizadas en el desarrollo de esta investigación.

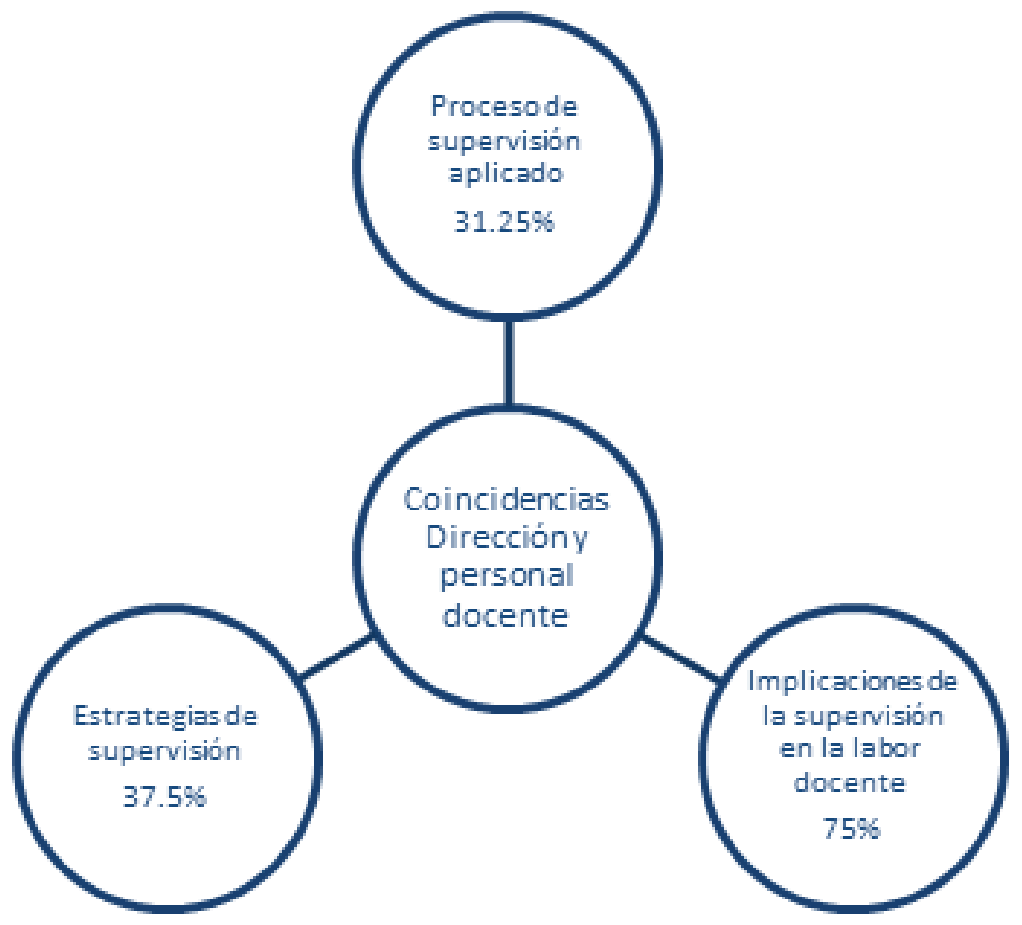

Figura 1. Coincidencias de respuestas entre el personal docente y la dirección del Colegio Técnico Profesional Ricardo Castro Beer.

Nota Elaboración propia a partir de la interpretación y análisis de la información suministrada por los sujetos participantes del estudio, 2012.

El personal docente y la dirección, en este caso, coinciden en un $75 \%$ de respuestas referentes a la categoría de implicaciones de la supervisión en la labor docente, lo que evidencia que si existe una interacción entre lo que se hace para supervisar y lo que se obtiene de su aplicación, en el ejercicio de las funciones docentes.

En relación con las estrategias de supervisión que aplica la dirección, concuerdan en un $37,5 \%$; lo que da a entender que si bien se llevan a cabo acciones de supervisión, éstas pueden mejorar y enriquecerse con nuevas técnicas y métodos al respecto. 
En un $31,25 \%$, el personal docente y la dirección coinciden en cuanto al proceso de supervisión que se lleva a cabo en el centro educativo, lo que manifiesta la ausencia de una acción supervisora plenamente planificada, seguida y evaluada de forma pertinente, para el mejoramiento del proceso de enseñanza aprendizaje y por ende, del quehacer educativo en la gestión escolar.

Para el CTP San Mateo, las coincidencias de opiniones entre el personal docente y la dirección, según las categorías analizadas, se presentan en la siguiente figura. La misma, muestra claramente una situación similar a la que se da en el CTP Ricardo Castro Beer, en la que prevalece el valor superior de coincidencia en la categoría que se relaciona con las implicaciones en la labor docente y el valor menor, en el proceso de supervisión que se realiza.

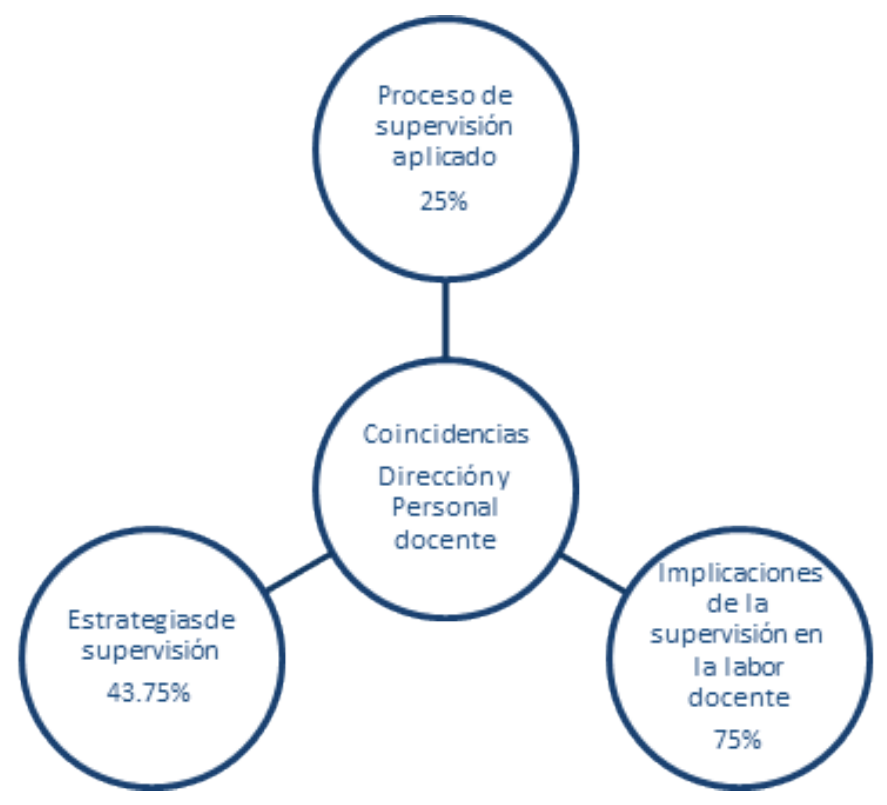

Figura 2. Coincidencias de respuestas entre el personal docente y la dirección del Colegio Técnico Profesional San Mateo.

Nota: Elaboración propia a partir de la interpretación y análisis de la información suministrada por los sujetos participantes del estudio, 2012. 
Así, en un 75\%, el personal docente y la dirección están de acuerdo en que las acciones supervisoras que se desarrollan, inciden plenamente en el cumplimiento de la labor docente. Lo que evidencia que la supervisión tiene injerencia directa en el desarrollo de funciones que desarrollan en relación con el proceso enseñanza y aprendizaje.

Para la categoría de estrategias de supervisión, las coincidencias marcan un 43,75\%, lo cual muestra que si se llevan a cabo acciones de inspección, orientación, asesoramiento y guía, en torno al desempeño docente. Estas acciones deben ampliarse y perfeccionarse, con el fin de aumentar la opinión docente y directiva, en más del cincuenta por ciento.

Por último, con un $25 \%$ de coincidencia aparece el proceso de supervisión que se lleva a cabo en el centro educativo, por lo que se considera que si bien se aplican técnicas de supervisión que inciden en las funciones docentes, éstas no están articuladas de manera secuencial y sistemática como un proceso.

De ahí, la necesidad, en ambas instituciones, de reforzar el proceso de supervisión, a partir de la consideración de las etapas que conforman el desarrollo oportuno de una supervisión, que se complemente con nuevas técnicas y procedimientos para el perfeccionamiento constante del desempeño profesional docente, el enriquecimiento de las relaciones interpersonales y la ejecución del proceso enseñanza aprendizaje.

\section{Propuesta: Herramientas de gestión para el desarrollo del proceso de supervisión de la labor del personal docente en las organizaciones educativas}

La presente propuesta responde a los resultados de la investigación realizada sobre las estrategias de supervisión que aplica la dirección en el Colegio Técnico Profesional Ricardo Castro Beer y el Colegio Técnico Profesional San Mateo, y sus implicaciones en el cumplimiento de labores del personal docente.

La justificación de la propuesta se sustenta de las principales conclusiones:

- Ambas instituciones reflejan una marcada ausencia de mecanismos de control. La información suministrada en los cuestionarios refleja debilidad en cuanto a la aplicación de instrumentos y técnicas de control durante el desarrollo de la supervisión. Se puede suponer que esta carencia de mecanismos de control es producto de no contar con un instrumento a la hora de realizar la visita de supervisión.

- Facilita la gestión administrativa. La gestión administrativa está llena de múltiplesfunciones que se realizan regular y rutinariamente, como de la atención de todos los imprevistos que surgen dentro de la comunidad educativa. Por lo que se considera prudente y oportuno brindar un instrumento que permita conocer la realidad del desempeño docente. 
- No permite subjetividad a la hora de realizar la supervisión. Por ser un ambiente de una alta interacción personal, surgen conflictos con gran facilidad, conflictos que pueden darse entre el personal, el director y el personal, entre otras líneas de comunicación. Si el administrador de la educación decide actuar con ética, a la hora de realizar su gestión administrativa en el campo de la supervisión, puede aplicar un instrumento y recogerá de ese funcionario que le ha causado problemas, la misma información de otros con los que lleva buena relación.

- Se puede archivar para obtener un perfil del desempeño del funcionario. Si se realiza una visita de supervisión por período o por año, y esta se archiva en el expediente institucional del funcionario, se podrá recopilar información histórica para comparar resultados. Lo que permite obtener la información requerida para felicitar, fortalecer o corregir aspectos en el desempeño del docente.

- Permite el conocimiento real y actual de la organización educativa en cuanto al desempeño docente. Al aplicar el instrumento de supervisión a varios docentes obtendrá información básica, por ejemplo: si detecta que el cumplimiento de los deberes de los docentes no es satisfactorio, deberá de analizar la imagen de respeto y de líder que emana. Brinda al administrador una oportunidad de autoevaluación de su gestión de la administración de la educación.

- Para la elaboración oportuna de un plan de mejora. La aplicación del instrumento de supervisión le permitirá evaluar, analizar y decidir las acciones a seguir para alcanzar la excelencia en la organización de la educación.

\section{Objetivos de la propuesta}

Para orientar el desarrollo de la propuesta, se establecen los siguientes objetivos:

- Diseñar herramientas que faciliten la gestión del proceso de supervisión de la labor docente en las organizaciones educativas.

- Definir los tipos de instrumentos requeridos por los profesionales en administración de la educación para llevar a cabo el proceso de supervisión de la labor docente.

- Determinar las categorías e indicadores requeridos para la elaboración de los instrumentos de supervisión sobre el desempeño de la labor docente.

\section{Desarrollo de la propuesta.}

La presente propuesta es un modelo funcional de gestión, a partir de herramientas diseñadas para el desarrollo del proceso de supervisión de la labor del personal docente en las 
organizaciones educativas, a partir de los principios que rigen la misma en la administración de la educación, en relación con los mecanismos de control para el cumplimiento de la labor docente.

El fin de la propuesta es brindar un aporte significativo a la acción supervisora del profesional en administración de la educación, a partir de un propósito claramente definido, que satisfaga la función a la cual responde.

\section{Plan de acción}

1. Definición del tipo de instrumento: Para llevar a cabo el proceso de supervisión, se proyecta la definición de tres tipos de instrumentos, en función de la acción supervisora que se desarrolle por parte del administrador de la educación y la población estudiantil. En ese sentido, se determina la entrevista estructurada para el personal docente y un cuestionario con escala para la opinión de los estudiantes, en relación con la labor docente en el aula, ambos aplicados por la dirección del centro educativo.

2. Entrevista estructurada al personal docente: Se selecciona la entrevista estructurada como instrumento para aplicar al personal docente, por considerarse de suma importancia la participación de los involucrados en el proceso de supervisión. Debido a que, se pretende facilitar la gestión supervisora de la labor docente, se construye un instrumento que consta de dos partes:

3. Recopilación de datos de los archivos: Antes de ir al aula a realizar la supervisión, el administrador debe recoger algunos datos del docente que deben constar en el expediente que el administrador debe tener de cada funcionario. Debe tener las fotocopias del instrumento que se aplicará a los estudiantes así como los lapiceros o lápices (para que después el supervisado no sienta tentación de investigar quién contesto, qué, por el color de tinta utilizado).

4. Criterios de supervisión de la labor docente: En el aula el administrador debe presentarse e iniciar el proceso de supervisión, para responder el instrumento el administrador deberá observar el grupo y la interacción del docente con el grupo y preguntar sobre aspectos relacionados con el proceso enseñanza aprendizaje, el cumplimiento de la labor docente, otros. A través de un proceso de comunicación se recopila información durante la entrevista, el entrevistado responde a cuestiones previamente diseñadas en función de los objetivos de la supervisión en presencia del entrevistador. Cabe destacar que en todo momento el administrador debe utilizar su conocimiento, experiencia, pericia responsabilidad y ética para modificar su plan de supervisión en caso de emerger un imprevisto.

5. Cuestionario con escala aplicado al grupo de estudiantes que se encuentran con el profesor supervisado en el momento. 


\section{Referencias}

Álvarez, M. y López, J. (1999). La evaluación del profesorado y de los equipos docentes. Madrid, España: Editorial Síntesis S.A.

Antúnez, S. (1993). Hacia una gestión autónoma del centro escolar. En: Claves para la organización de centros escolares. Barcelona: ICE/Horsori.

Arroyo, J. (2000). Administración estratégica en las instituciones educativas, innovación y cambio. San José, Costa Rica: UCR-MEP.

Batlle, F. (2010). Acompañamiento docente como herramienta de construcción. Revista Electrónica de Humanidades, Educación y Comunicación Social, 5 (8), pp. 102-110. Recuperado en: http:// www.publicaciones.urbe.edu/index.php/REDHECS/article/view/580/1457

Blejmar, B. (2005). Gestionar es hacer que las cosas sucedan. Buenos Aires, Argentina: Ediciones Novedades Educativas.

Cambronero, M. (2002). Algunos factores de la supervisión educativa que influyen en la calidad del proceso enseñanza aprendizaje en la Escuela Jacinto Ávila Araya, Palmares, Alajuela, Costa Rica. (Informe de Seminario Proyecto de Graduación correspondiente al Plan de Estudios de Maestría en Administración de la Educación). Recuperado en: www.uned.ac.cr/SEP/ recursos/investigaciones/documents/maria_marta.pdf.

Campos A., R. (2008). Supervisión educativa. Oficina Diocesana de Educación Católica de Chimbote. Recuperado en: http://www.buenastareas.com/ensayos/Supervision-Educativa/2596241.html

Chiavenato, I. (2006). Introducción a la teoría general de la administración. México: McGraw Hill.

Dirección Servicio Civil. (2004). Manual descriptivo de clases de puestos docentes. Recuperado en: http://www.mep.go.cr/CentroDelnformacion/DOC/9\%20Manual\%20Descriptivo\%20 de\%20Clases\%20de\%20Puestos\%20Docentes-30320098487.pdf 
Garbanzo, G. (2002). La supervisión educativa: eje para el desarrollo de maestros y maestras, profesores y profesoras eficaces del sistema educativo costarricense: un enfoque en busca de la calidad educativa. Universidad de Costa Rica. San José, Costa Rica.

García, M.; Rojas, N. y Campos, N. (2002). La administración escolar. San José, Costa Rica: Editorial de la Universidad de Costa Rica.

Gento, S. (2008). La gestión y supervisión de centros educativos. San José, Costa Rica: EUNED.

Gutiérrez, R. V. (2006). Administración. Colombia: Editorial: Thomson Learning.

Fernández, M. y Gutiérrez, M. (2005).Organización escolar, profesión docente y entorno comunitario. Madrid, España: Ediciones AKAL, S.A.

Hernández, R., Fernández, C. y Baptista, P. (2010). Metodología de la investigación. México: McGraw Hill.

López, A. (2011). Relaciones interpersonales. Recuperado en: http://www.slideshare.net/ AuraLopez3/relaciones-interpersonales-14702584

Robbins, S. (2004).Comportamiento organizacional. México: PEARSON EDUCACION.

Rodríguez, J. (2006). El sistema de dirección en la organización. México: CENGAGE LEARNING.

Romero, F. (2001). Supervisión y evaluación de centros educativos. Programa Nacional de Desarrollo de Administradores Educativos (PRONDAE), Ministerio de Educación Pública (MEP). San José, Costa Rica: Departamento de Publicaciones MEP.

Teixidó, M. (1997). Supervisión del sistema educativo. España: Ariel S.A.

Venegas, P. (2000). Principios, fuentes y referencias de la administración educativa moderna. San José, Costa Rica: CENADI. 